\title{
BMJ Open APpropriAteness of percutaneous Coronary interventions in patients with ischaemic HEart disease in Italy: the APACHE pilot study
}

\author{
Sergio Leonardi, ${ }^{1}$ Marcello Marino, ${ }^{2}$ Gabriele Crimi, ${ }^{1}$ Florinda Maiorana, ${ }^{1}$ \\ Diego Rizzotti, ${ }^{1}$ Corrado Lettieri, ${ }^{3}$ Luca Bettari, ${ }^{4}$ Marco Zuccari, ${ }^{5}$ Paolo Sganzerla, ${ }^{6}$ \\ Simone Tresoldi, ${ }^{7}$ Marianna Adamo, ${ }^{8}$ Sergio Ghiringhelli, ${ }^{9}$ Carlo Sponzilli, ${ }^{10}$ \\ Giampaolo Pasquetto, ${ }^{11}$ Andrea Pavei, ${ }^{12}$ Luigi Pedon, ${ }^{13}$ Luciano Bassan, ${ }^{14}$ \\ Mario Bollati, ${ }^{15}$ Paola Camisasca, ${ }^{16}$ Daniela Trabattoni, ${ }^{17}$ Marta Brancati, ${ }^{18}$ \\ Arnaldo Poli, ${ }^{19}$ Claudio Panciroli, ${ }^{20}$ Maddalena Lettino, ${ }^{21}$ Giuseppe Tarelli, ${ }^{21}$ \\ Giuseppe Tarantini, ${ }^{22}$ Leonardo De Luca, ${ }^{23}$ Ferdinando Varbella, ${ }^{24}$ \\ Giuseppe Musumeci, ${ }^{25}$ Stefano De Servi ${ }^{26}$
}

To cite: Leonardi S, Marino M, Crimi G, et al. APpropriAteness of percutaneous Coronary interventions in patients with ischaemic HEart disease in Italy: the APACHE pilot study. BMJ Open 2017;7:e016909. doi:10.1136/ bmjopen-2017-016909

- Prepublication history and additional material for this paper are available online. To view these files, please visit the journal online (http://dx.doi. org/10.1136/bmjopen-2017016909).

Received 20 March 2017 Revised 26 July 2017 Accepted 4 August 2017

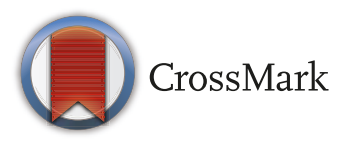

For numbered affiliations see end of article.

Correspondence to

Dr Sergio Leonardi;

s.leonardi@smatteo.pv.it

\section{ABSTRACT}

Objectives To first explore in Italy appropriateness of indication, adherence to guideline recommendations and mode of selection for coronary revascularisation.

Design Retrospective, pilot study.

Setting 22 percutaneous coronary intervention (PCl)performing hospitals (20 patients per site), 13 (59\%) with on-site cardiac surgery.

Participants 440 patients who received PCl for stable coronary artery disease (CAD) or non-ST elevation acute coronary syndrome were independently selected in a 4:1 ratio with half diabetics.

Primary and secondary outcome measures Proportion of patients who received appropriate $\mathrm{PCl}$ using validated appropriate use scores (ie, AUS $\geq 7$ ). Also, in patients with stable CAD, we examined adherence to the following European Society of Cardiology recommendations: (A) per cent of patients with complex coronary anatomy treated after heart team discussion; (B) per cent of fractional flow reserve-guided $\mathrm{PCl}$ for borderline stenoses in patients without documented ischaemia; (C) per cent of patients receiving guideline-directed medical therapy at the time of $\mathrm{PCl}$ as well as use of provocative test of ischaemia according to pretest probability (PTP) of CAD.

Results Of the 401 mappable PCls (91\%), 38.7\% (95\% Cl 33.9 to 43.6$)$ were classified as appropriate, $47.6 \%(95 \%$ Cl 42.7 to 52.6) as uncertain and $13.7 \%$ (95\% Cl 10.5\% to $17.5 \%$ ) as inappropriate. Median PTP in patients with stable CAD without known coronary anatomy was $69 \%$ (78\% intermediate PTP, 22\% high PTP). Ischaemia testing use was similar $(p=0.71)$ in patients with intermediate $(n=140,63 \%)$ and with high PTP $(n=40,66 \%)$. In patients with stable CAD ( $n=352)$ guideline adherence to the three recommendations explored was: (A) 11\%; (B) 25\%; (C) $23 \%$. AUS was higher in patients evaluated by the heart team as compared with patients who were not (7 (6.8) vs 5 (4.7); $p=0.001$ ).

Conclusions Use of heart team approaches and adherence to guideline recommendations on coronary
Strengths and limitations of this study

APACHE (APpropriAteness of percutaneous Coronary interventions in patients with ischaemic HEart disease) is a first-in-class study in Italy designed to measure the degree of appropriateness of indication, multidisciplinary decision-making processes and implementation of key guideline recommendations in patients undergoing percutaneous coronary intervention $(\mathrm{PCl})$.

- This study, which enrolled patients with stable coronary artery disease and diabetes, was intentionally designed to focus on high-risk patients for inappropriate $\mathrm{PCl}$. Therefore, true appropriateness of $\mathrm{PCl}$ may be underestimated. However, rather than an epidemiological study, APACHE's intention was to serve as first initiative sponsored by a national medical society to measure care process and improve quality.

- APACHE examined the appropriateness of $\mathrm{PCl}$ indication, not of coronary angiography. Therefore, we acknowledge as a limitation that we have no data to inform appropriateness of surgical revascularisation, nor indication to invasive angiography.

revascularisation in a real-world setting is limited. This pilot study documents the feasibility of measuring appropriateness and guideline adherence in clinical practice and identifies substantial opportunities for quality improvement.

Trial registration number NCT02748603.

\section{INTRODUCTION}

Percutaneous coronary intervention (PCI) has dramatically improved the prognosis of 
patients with coronary artery disease (CAD). Yet, many patients receive PCI whose clinical indication appears uncertain or inappropriate, especially in the non-acute setting. ${ }^{2}$ The development of appropriate use criteria by cardiovascular societies has provided the basis for a standardised approach to systematically assess the clinical appropriateness of $\mathrm{PCI}^{3}$ and has produced a reduction in the volume of non-acute PCI as well as an increase in the proportion of procedures classified as appropriate, ${ }^{4}$ but these studies have been mostly performed in the USA.

In Europe, data on appropriateness of indication and mode of selection for coronary revascularisation strategies as well as the degree of implementation of guideline recommendations are limited. ${ }^{5}$ European Society of Cardiology (ESC) guidelines ${ }^{67}$ urge the implementation of a multidisciplinary decision-making approach-the heart team-to select the optimal mode of revascularisation, but data on the implementation of this process in patients with complex $\mathrm{CAD}$, including those with stable CAD and diabetes, are scarce. ${ }^{8}$ Specifically, the ESC $^{67}$ recommends that (1) complex pathologies in stable patients, including lesions of the left main or proximal left anterior descending artery (LAD) and threevessel disease, should in general not be treated ad hoc, but discussed by the heart team; (2) pressure-derived fractional flow reserve (FFR) should be used to identify haemodynamically relevant coronary lesion(s) in stable patients when evidence of ischaemia is not available; and (3) patients with stable CAD must receive guideline-recommended medical treatment prior to revascularisation.

We designed the APACHE (APpropriAteness of percutaneous Coronary interventions in patients with ischaemic HEart disease) pilot study to first explore the degree of appropriateness of indication of PCI, multidisciplinary decision-making processes and implementation of key guideline recommendations in patients undergoing PCI in Italy.

\section{METHODS}

\section{Study design and patient selection}

APACHE was designed as a pilot initiative to assess appropriateness of PCI indication and adherence to key guideline recommendations on coronary revascularisation in patients with predominantly stable $\mathrm{CAD}$ and diabetes, considered to be at high risk for inappropriate indication and mode for coronary revascularisation (ie, PCI treatment in patients with an indication for CABG). All PCI-performing hospitals of the Lombardia and Veneto regions in Italy, serving a population of $\approx 15000000$ people, were invited to participate in the study. Twenty-two sites agreed to participate, obtained regulatory approval and were eventually included.

At each participating hospital, 20 patients were independently selected on-site by the study team (see online supplementary appendix) among consecutive patients who were admitted in the previous year for an elective procedure to treat stable $\mathrm{CAD}$ or urgently for an episode of non-ST elevation acute coronary syndrome (NSTEACS) in a 4:1 ratio without site personnel involvement in the selection of the cases identified to minimise selection bias. The study population was also selected to preserve an overall 1:1 ratio on diabetes status. If the number of patients selected was insufficient, older cases were evaluated for possible inclusion. Due to the low likelihood of receiving a redo procedure as well as the inability to measure a SYNTAX (SYNergy between percutaneous coronary intervention with TAXus and cardiac surgery) score, patients with a history of bypass surgery were excluded.

\section{Data collection, core angiographic assessment and central heart team}

Variables of interest were collected by the study team during dedicated visits at participating hospitals via clinical chart abstraction. Sites were requested to provide the complete clinical chart, including the coronary angiogram of the index PCI. Source documentation was reviewed in full to abstract symptoms status (angina class); cardiovascular risk factors and comorbidities; medical therapy at time of PCI; site-reported indication for PCI; presence, results and timing of any non-invasive functional test, FFR or intracoronary imaging, if performed; coronary anatomy and reported significance of angiographic stenoses for treated lesion(s) on the catheterisation report; and evidence for heart team discussion involving a cardiac surgeon. Finally, pretest probability (PTP) of significant CAD was calculated in patients with stable CAD according to guideline recommendations. ${ }^{7}$

The Angiographic Core Laboratory (ACL) was composed of two independent physicians with experience in interventional cardiology (MM, GC) who centrally and independently reviewed coronary angiography for each patient to define (1) baseline SYNTAX score, (2) category of coronary anatomy (eg, one, two or threevessel $\mathrm{CAD}$ with or without proximal $\mathrm{LAD}$ involvement) ${ }^{3}$ and (3) presence of 'borderline' angiographic stenoses $(50 \%-60 \%)$. For the SYNTAX score, a disagreement was arbitrarily considered to be present if there was a between score difference $\geq 10$ or both scores were not in the same tertile $(0-22,23-32,>32)$. In case of agreement, an average SYNTAX score was calculated. In case of disagreement between reviewers, the case was first resolved by consensus. If a consensus could not be reached (or if the case was deemed particularly challenging), the conflict was resolved by the central heart team. Anatomical category and presence of borderline coronary stenoses were analysed by a single reviewer (GC or MM).

The central heart team was represented by four members-two interventional cardiologists (LDL, FV), one cardiac surgeon (GT) and one clinical cardiologist (ML) - nominated by the Italian Society of Interventional Cardiology (SICI-GISE) among recognised experts in their respective specialty. The role of the central heart team was to review cases with unresolved conflicts by the ACL, cases considered complex or challenging by the 
study team or cases with incomplete or conflicting documentation. Assessments of the central heart team were performed by consensus.

\section{Evaluation of appropriateness of indications for coronary revascularisation}

The comprehensive documentation of indications for PCI was formally examined based on the 2013 ACC/ AHA/SCAI/AMA-PCPI/NCQA PCI measurement set. ${ }^{9}$ This diagnostic measure was defined as the proportion of patients whose clinical documentation includes, at a minimum, the following elements: (1) priority (acute coronary syndrome, elective, urgent, emergency/salvage); (2) presence and severity of angina symptoms (eg, Canadian Cardiovascular Society classification system); (3) use of antianginal medical therapies within 2 weeks before the procedure, if any; (4) presence, results and timing of non-invasive stress test, FFR or intravascular ultrasound, if performed; and (5) significance of angiographic stenosis on coronary angiography for treated lesion.

Appropriateness of indication of coronary revascularisation was examined by assigning to each procedure an appropriate use score (AUS) and corresponding criteria (AUC, appropriate use criteria), with a score of 1 indicating a completely inappropriate procedure to a score of 9 indicating a completely appropriate one. ${ }^{3}$ Scores of 7-9 indicate that revascularisation is considered generally appropriate and likely to improve patients' symptoms or survival. Scores of 1-3 are considered generally inappropriate while scores of 4-6 indicate a clinical scenario for which the likelihood that coronary revascularisation will improve health outcomes or symptoms is uncertain. This score was defined by considering clinical presentation; severity of angina; extent of ischaemia on non-invasive testing; presence of other prognostic factors, such as congestive heart failure or depressed left ventricular function; extent of medical therapy at the time of PCI; and extent of anatomic coronary disease. If the scenario was not considered by the consensus document, ${ }^{3}$ the procedure was considered non-mappable.

To limit site operator-related bias, the study team calculated two scores for each procedure:

1. $\mathrm{AUS}_{\mathrm{SITE}}$, based on site-reported extent of anatomic coronary disease;

2. $\mathrm{AUS}_{\mathrm{CORE}}$, based on ACL-reported extent of anatomic coronary disease.

\section{Evaluation of adherence to ESC guidelines and heart team processes}

In patients with stable $\mathrm{CAD}$, we assessed adherence to three class I recommendations according to ESC guidelines ${ }^{67}$ :

\section{Recommendation 1}

Proportion of patients with stable $\mathrm{CAD}$ and complex anatomy (including lesions of the left main, proximal $\mathrm{LAD}$ and/or three-vessel CAD) who were treated after local heart team discussion. This recommendation was explored using both site-reported and ACL-reported coronary anatomy. We also explored adherence to this recommendation by calculating the proportion of patients with complex anatomy who received ad hoc PGI without documented heart team discussion. To better define the optimal mode of coronary revascularisation, the SYNTAX II score ${ }^{10}$ as well as the Society of Thoracic Surgeons (STS) score, ${ }^{11}$ and EuroScore II $^{12}$ were calculated.

\section{Recommendation 2}

Proportion of patients with stable CAD, no evidence of ischaemia and borderline lesions according to the ACL in whom FFR was used to identify haemodynamically relevant coronary lesion (s).

Specifically, this recommendation was explored as follows:

(A) proportion of patients with no functional test (ie, test negative or not performed) and at least one borderline stenosis according to ACL in whom pressure-derived FFR was used; (B) proportion of patients with no functional test or asymptomatic and at least one borderline stenosis according to ACL in whom pressure-derived FFR was used; (C) proportion of patients with no functional test or asymptomatic and site-reported multivessel CAD in whom pressure-derived FFR was used. ${ }^{13}$

\section{Recommendation 3}

Proportion of patients with stable CAD who received guideline-directed medical therapy prior to revascularisation. $^{7}$

Specifically: (A) proportion of patients without known allergy or documented intolerance taking low-dose aspirin (75-150 mg daily) or clopidogrel; (B) proportion of patients without known allergy or documented intolerance receiving a statin; (C) proportion of patients with heart failure, hypertension or diabetes treated with an ACE inhibitor or angiotensin receptor blocker; (D) proportion of patients on optimal medical therapy defined as drugs for event prevention (aspirin and/ or clopidogrel; a statin; an ACE-Inhibitor/Angiotensin Receptor Blocker if heart failure, hypertension or diabetes) plus at least one drug for angina relief if symptomatic, such as beta blockers, calcium channel blockers, long-acting nitrates, ivabradine or ranolazine.

In patients with NSTEACS, we examined the proportion of PCI procedures performed within 24hours of admission in patients with a GRACE (Global Registry of Acute Coronary Events) score $>140$.

Finally, we assessed, by structured investigators' surveys, the presence of written institutional protocols developed locally by the heart team in accordance with current guidelines including specific anatomical criteria and clinical subsets that may be (or should not be) treated ad hoc as well as the modalities and timing for convocation of heart team meetings. 


\section{Statistical analysis and sample size considerations}

Categorical data are presented as counts and proportion and continuous data as median (25th, 75 th percentiles) and were analysed, as appropriate, using $\chi^{2}$ test (or Fisher's exact test) and Wilcoxon rank-sum test. We calculated 95\% CIs for the proportion using the normal approximation to the binomial calculation. To control for the effect of participating site as well as the presence of cardiac surgery on-site on the primary outcome (AUS), we used analysis of variance provided in the generalised linear model procedure of SPSS V.20 including participating site and presence of cardiac surgery on-site as cofactors for the key subgroup (factor) of interest (ie, patients evaluated by the heart team vs not).

Given the lack of prior studies to estimate appropriateness of coronary revascularisation in Italy, sample size estimation was challenging. The study was powered on the primary subgroup of interest, patients with stable CAD, assuming an appropriateness of $35 \%$ in this patient population based on prior reports. ${ }^{4}$ Using a normal approximation to the binomial distribution for this proportion, a population of 350 patients with stable CAD was needed to obtain a $95 \%$ CI between $30 \%$ and $40 \%$ for appropriateness. ${ }^{14}$ Analyses were performed using SPSS V.20 and sample size was estimated using http:/ /www.samplesize.net/. The study obtained institutional review board approval by all participating hospitals and is registered on ClinicalTrial.Gov ID: NCT02748603.

\section{Sponsor and funding}

The APACHE study was designed by the chair and principal investigator and approved by the institutional review board at each participating centre. The study was sponsored by the Italian Society of Invasive Cardiology (SICI-GISE), a non-profit organisation, and received unrestricted grant support from the Abbott Vascular and Daiichi-Sankyo. The sponsor and funders had no role in the design of the study, the collection, monitoring, analysis and interpretation of the data, or the writing of the report. The first draft of the manuscript was written by the first author. All the authors vouch for the accuracy and completeness of the data and all analyses.

\section{RESULTS}

Of the 22 hospitals included, 13 (59\%) have on-site cardiac surgery and $4(18 \%)$ are private hospitals. Overall, PCI procedures of 440 patients (performed between January 2014 and May 2016) were included: 352 for patients with stable CAD and 88 with NSTEACS (12 with unstable angina and 76 with non-ST elevation myocardial infarction; median GRACE score 109 (89.5, 125.5), median CRUSADE (Can Rapid risk stratification of Unstable angina patients Suppress ADverse outcomes with Early implementation of the ACC/AHA guidelines) score 24 $(16,40))$. A SYNTAX score could be calculated in 422 patients $(96 \%)$ with 87 disagreements $(21 \%)$ between ACL reviewers. Of these, 55 were resolved by consensus and 32 by the central heart team. Clinical profile of the selected patients stratified by clinical indication is shown in table 1. By design, almost half of the patients had diabetes $(n=216,49.1 \%)$ with a high proportion of patients with dyslipidaemia (54\%), prior PCI $(40 \%)$ and history of angina $(69 \%)$. Table 2 presents data on indication and test selection in patients with stable CAD. Median PTP of CAD in patients with stable CAD without known coronary anatomy was 69\% (78\% intermediate PTP, 22\% high PTP, no patient with low PTP). The use of provocative tests of ischaemia was similar $(\mathrm{p}=0.71)$ in patients with intermediate PTP $(\mathrm{n}=140,63 \%)$ and with high PTP $(\mathrm{n}=40,66 \%)$. Of the 88 patients with NSTEACS, a GRACE score $>140$ was present in $11(12 \%)$ patients. Of these, five patients had PCI within 24 hours.

Two of the secondary outcomes listed on ClinicalTrials. gov, that is, (1) proportion of patients receiving incomplete revascularisation (ie, residual SYNTAX $>8$ ) and (2) proportion of patients with NSTEACS who are stabilised (no recurrent ischaemic symptoms), who have multivessel disease and a high SYNTAX score ( $>22)$, without documentation of heart team discussion in the medical records for NSTEACS, were not considered due to the limited reproducibility observed for the SYNTAX score and the small number of subjects $(n=3)$, respectively.

\section{Comprehensive documentation and appropriateness of indications for coronary revascularisation}

A comprehensive documentation of PCI indication was present in 427 (97\%) patients. Most common reasons for unfulfilling this diagnostic measure were the lack of documentation in the clinical chart of any non-invasive testing, both functional and imaging $(n=5)$, or missing information on therapy at admission $(n=8)$.

An AUS $_{\text {SITE }}$ could be calculated in 405 (92\%) of patients while the remaining 35 patients did not have comprehensive documentation of indications for PCI or the scenario was not applicable (ie, non-mappable AUC). The median AUS $_{\text {SITE }}$ was 6 (5.7) corresponding to $153(37.8 \%, 95 \%$ CI 33.1 to 43.5$)$ of PCI classified as appropriate, 193 (47.7\%, $95 \% \mathrm{CI} 42.8$ to 52.5$)$ as uncertain and $59(14.6 \%, 95 \% \mathrm{CI}$ 11.1 to 18) as inappropriate AUC, similar to patients with and without diabetes (figure 1 and figure 2). $\mathrm{AUS}_{\text {SITE }}$ was higher in patients evaluated by the local heart team as compared with patients who were not, both in unadjusted (7 (5.8) vs 5 (4.7); $\mathrm{p}=0.003$ ) and adjusted analyses (mean AUS $_{\text {SITE }} 7.0$, SD 1.8 vs 5.7, SD 1.9; $\mathrm{p}=0.001$; participating site and cardiac surgery on-site both non-significant).

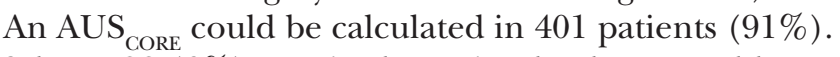
Of these, $23(6 \%)$ required a review by the central heart team. Median $\mathrm{AUS}_{\mathrm{CORE}}$ was 6 (5.7) with 155 (38.7\%, 95\% CI 33.9 to 43.4 ) of PCI classified as appropriate, $191(47.6 \%, 95 \%$ CI 42.7 to 52.5$)$ as uncertain and 55 (13.7\%, 95\% CI 10.3 to 17.1) as inappropriate AUC. AUS $_{\mathrm{CORE}}$ results by site and in key subgroups are reported in figure 2 and the online supplementary figure, respectively. $\mathrm{AUS}_{\mathrm{CORE}}$ was higher in patients with NSTEACS as compared with patients with stable CAD with no 
Table 1 Baseline characteristics, cardiovascular risk factors and history stratified by clinical indication

\begin{tabular}{|c|c|c|c|}
\hline & Stable CAD $(n=352)$ & NSTEACS $(n=88)$ & Overall $(n=440)$ \\
\hline Age (years) & $69.3(62.9-75.1)$ & $71(63.4-77.4)$ & $69.6(63-75.8)$ \\
\hline Female, n (\%) & $71(20)$ & $27(31)$ & $98(22)$ \\
\hline Creatinine (mg/dL) & $0.9(0.79,1.08)$ & $0.97(0.85,1.18)$ & $0.91(0.8,1.10)$ \\
\hline SYNTAX score & $12(8-20)$ & $15(8-20)$ & $13(8-20)$ \\
\hline Diabetes, n (\%) & $173(49)$ & $43(49)$ & $216(49)$ \\
\hline Hypertension, n (\%) & $262(75)$ & $71(78)$ & $333(75)$ \\
\hline Dyslipidaemia, n (\%) & $190(54)$ & $48(55)$ & $238(54)$ \\
\hline Active smoker, n (\%) & $53(15)$ & $16(18)$ & $69(16)$ \\
\hline Prior smoker, n (\%) & $85(24)$ & $13(15)$ & $98(22)$ \\
\hline Prior MI, n (\%) & $95(27)$ & $20(23)$ & $115(26)$ \\
\hline Prior PCI, n (\%) & $148(42)$ & $28(32)$ & $176(40)$ \\
\hline Renal insufficiency, n (\%) & $38(11)$ & $20(23)$ & $58(13)$ \\
\hline Heart failure, n (\%) & $14(4)$ & $2(2)$ & $16(4)$ \\
\hline LVSD, n (\%) & $23(7)$ & $8(9)$ & $31(7)$ \\
\hline COPD, n (\%) & $26(7)$ & $8(9)$ & $34(8)$ \\
\hline Stroke, n (\%) & $18(5)$ & $5(6)$ & $23(5)$ \\
\hline PAD, n (\%) & $55(16)$ & $10(11)$ & $65(15)$ \\
\hline
\end{tabular}

BMI, body mass index; CAD, coronary artery disease; COPD, chronic obstructive pulmonary disease; CV, cardiovascular; LVSD, left ventricular systolic dysfunction defined as ejection fraction of 0.40 or less; MI, myocardial infarction; NSTEACS, non-ST elevation acute coronary syndrome; PAD, peripheral artery disease; PCI, percutaneous coronary intervention; SYNTAX, SYNergy between percutaneous coronary intervention with TAXus and cardiac surgery.

significant difference according to diabetic status or type of hospitals. $\mathrm{AUS}_{\mathrm{CORE}}$ was higher in patients evaluated by the local heart team as compared with patients who were not both in unadjusted (7 (6.8) vs $5(4.7) ; \mathrm{p}=0.001)$ and adjusted analyses (mean AUS $_{\text {CORE }} 7.1$, SD 1.5 vs 5.8, SD $1.9 ; \mathrm{p}=0.001$; participating site and cardiac surgery on-site both non-significant) (figure 3).

Recommendation 1: proportion of patients with stable CAD with complex pathologies who were treated after heart team discussion Of the 352 patients with stable CAD, 148 (42\%) had a complex site-reported coronary anatomy including significant lesions of the left main $(n=16)$, proximal LAD $(n=73)$ and three-vessel disease $(n=59)$. Of these, 17 $(11 \%)$ underwent local heart team discussion. Median operative mortality was low to intermediate as estimated by both the EuroScore II $(1.15 \%(0.64,2.05))$ and the STS score $(0.92 \%(0.45,1.81))$. Also, 118 of the 148 patients with complex site-reported coronary anatomy $(80 \%)$ received ad hoc PCI without evidence of discussion with the local heart team in the clinical chart, with no difference in patients treated at hospitals with or without on-site cardiac surgery $(p=0.74)$. The proportion of patients with complex coronary lesions according to the ACL was 46\% (n=164). Of these, $20(12 \%)$ underwent local heart team discussion and $124(75 \%)$ were treated ad hoc without evidence of heart team discussion.

The median SYNTAX score in patients with stable CAD was $12(8-20)$, with $83 \%$ of patients with a score $<23,13 \%$ between 23 and 32, and 4\% above 32 .

A SYNTAX II score could be calculated in 337 cases $(96 \%)$. Of these, CABG was the recommended option for 40 patients (12\%), PCI was the recommended option for $14(4 \%)$ and either mode of revascularisation was recommended in the remaining 283 cases (84\%). Of the 40 patients where SYNTAX II score recommended CABG, a local heart team discussion was performed in three cases (7\%). Finally, a total of 75 patients with stable CAD (21\%) with diabetes and multivessel CAD underwent ad hoc PCI without local heart team discussion documented in the patient's chart.

Recommendation 2: proportion of patients with stable CAD with no evidence of ischaemia where pressure-derived FFR was used to identify haemodynamically relevant coronary lesion(s)

Of the 352 patients with stable CAD, 151 (43\%) had no objective evidence of ischaemia (135 patients had 
Table 2 Characterisation of the indication for $\mathrm{PCl}$ in patients with stable CAD $(n=352)$

\begin{tabular}{|c|c|}
\hline Parameter & Value \\
\hline Pretest probability of CAD $(\%)^{*}$ & $69(54-84)$ \\
\hline Low (<15\%), n (\%) & $0(0)$ \\
\hline Intermediate (15\%-85\%), n (\%) & $222(78)$ \\
\hline High (>85\%), n (\%) & $61(22)$ \\
\hline Ad hoc PCl, n (\%) & $307(87)$ \\
\hline $\begin{array}{l}\text { Any functional test of ischaemia performed, } n \\
(\%) \dagger\end{array}$ & $217(62)$ \\
\hline Exercise ECG, n & 153 \\
\hline SPECT, $n$ & 46 \\
\hline Stress echocardiography, $\mathrm{n}$ & 31 \\
\hline Stress cardiac MRI, n & 3 \\
\hline Coronary CT angiography, n (\%) & $27(8)$ \\
\hline No functional or anatomical testing, n (\%) & $125(35 \%)$ \\
\hline $\mathrm{AUC}_{\text {CORE }}$ mappable & $318(90)$ \\
\hline Appropriate, n (\%) & $102(32)$ \\
\hline Uncertain, n (\%) & $163(51)$ \\
\hline Inappropriate, n (\%) & $52(16)$ \\
\hline $\mathrm{AUC}_{\text {SITE }}$ mappable & $320(91)$ \\
\hline Appropriate, n (\%) & $100(31)$ \\
\hline Uncertain, n (\%) & $163(51)$ \\
\hline Inappropriate, n (\%) & $57(18)$ \\
\hline
\end{tabular}

${ }^{*}$ Calculated according to (5) only in patients with unknown coronary anatomy $(n=285)$ : defined as a history of invasive coronary angiography or coronary CT angiography in the year preceding the index $\mathrm{PCl}$.

†Some patients $(n=15)$ underwent more than one functional test before $\mathrm{PCl}$; one patient received three tests.

$\mathrm{CAD}$, coronary artery disease; $\mathrm{PCl}$, percutaneous coronary intervention; SPECT, single-photon emission CT, AUC: Appropriate Use Criteria

no provocative test of ischaemia, 8 had negative testing and 8 had inconclusive testing), $36(10 \%)$ were asymptomatic and $82(23 \%)$ had multivessel CAD according to the site. A pressure-derived FFR to guide PCI was used for 29 patients $(8.2 \%)$ with stable CAD while intravascular ultrasound was used for 12 patients $(3 \%)$. No PCI was guided by coronary optical coherence tomography.

Of the 151 patients with no evidence of ischaemia, the ACL identified 28 patients $(18 \%)$ with at least one borderline coronary lesion treated with PCI with FFR performed in 7 of these 28 cases $(25 \%)$.

Of the $175(50 \%)$ patients who had no objective evidence of ischaemia or were asymptomatic, 33 borderline lesions were identified by the ACL with FFR performed in 10 cases. In the subgroup of 91 patients who also had site-reported multivessel CAD, 13 borderline lesions were identified by the ACL, with FFR performed in 4 cases.
Recommendation 3: use of guideline-directed medical therapy at the time of $\mathrm{PCl}$ in patients with stable $\mathrm{CAD}$

Of the 352 patients with stable CAD, 299 (85\%) were treated with single antiplatelet therapy at the time of angiography (292 with low-dose aspirin and 7 with clopidogrel) and $20(6 \%)$ received dual antiplatelet therapy with aspirin and clopidogrel (only 1 reported case of aspirin intolerance who successfully underwent aspirin desensitisation); 266 (76\%) were on a statin (no reported case of statin intolerance); $202(57 \%)$ received an ACE-I or an angiotensin receptor blocker (no reported case of allergy or intolerance). Among the subgroup of patients with stable CAD with hypertension, heart failure (or asymptomatic left ventricular ejection fraction of $40 \%$ or less) or diabetes $(\mathrm{n}=265 ; 75 \%$ of the overall stable CAD population), a treatment with ACE-I or ARB was given to 176 patients $(66 \%)$. Finally, a therapy with an antianginal agent (beta blocker, calcium channel blocker, nitrates, ivabradine or ranolazine) was administered to 237 patients overall and to 169 patients of the 248 with symptoms of angina $(68 \%)$. Overall, a total of 100 patients $(23 \%)$ received guideline-directed medical therapy at time of PCI.

\section{Adoption of institutional heart team protocols}

Investigators from all participating centres $(n=22)$ were interviewed and all responded. A written institutional heart team protocol was available in five $(23 \%)$ centres (one with on-site cardiac surgery, four without on-site cardiac surgery) while in other five centres (three with on-site cardiac surgery) heart team meetings were being scheduled on a regular basis (usually weekly). All other hospitals $(n=12)$ did not have either a heart team institutional protocol or regularly planned heart team meetings.

\section{DISCUSSION}

In this pilot investigation, we assessed appropriateness of indication of coronary revascularisation and adherence to key guideline recommendations in a real-world population with a high prevalence of stable CAD and diabetes as well as multidisciplinary decision-making processes. We identified important gaps in implementations of guideline recommendations and opportunities to improve the care of patients undergoing PCI.

\section{Considerations on appropriateness of indication for coronary revascularisation}

The proportion of appropriate indication for coronary revascularisation was $39 \%$ in the overall population and $32 \%$ in patients with stable CAD with similar rates observed using local versus ACL anatomical category. These proportions are similar to what was observed in the USA when AUC was first released in 2009. ${ }^{415}$ As expected, appropriateness was higher in patients with NSTEACS as compared with patients with stable CAD but similar according to diabetes status as well as in hospitals with and without on-site cardiac surgery. Importantly, both 


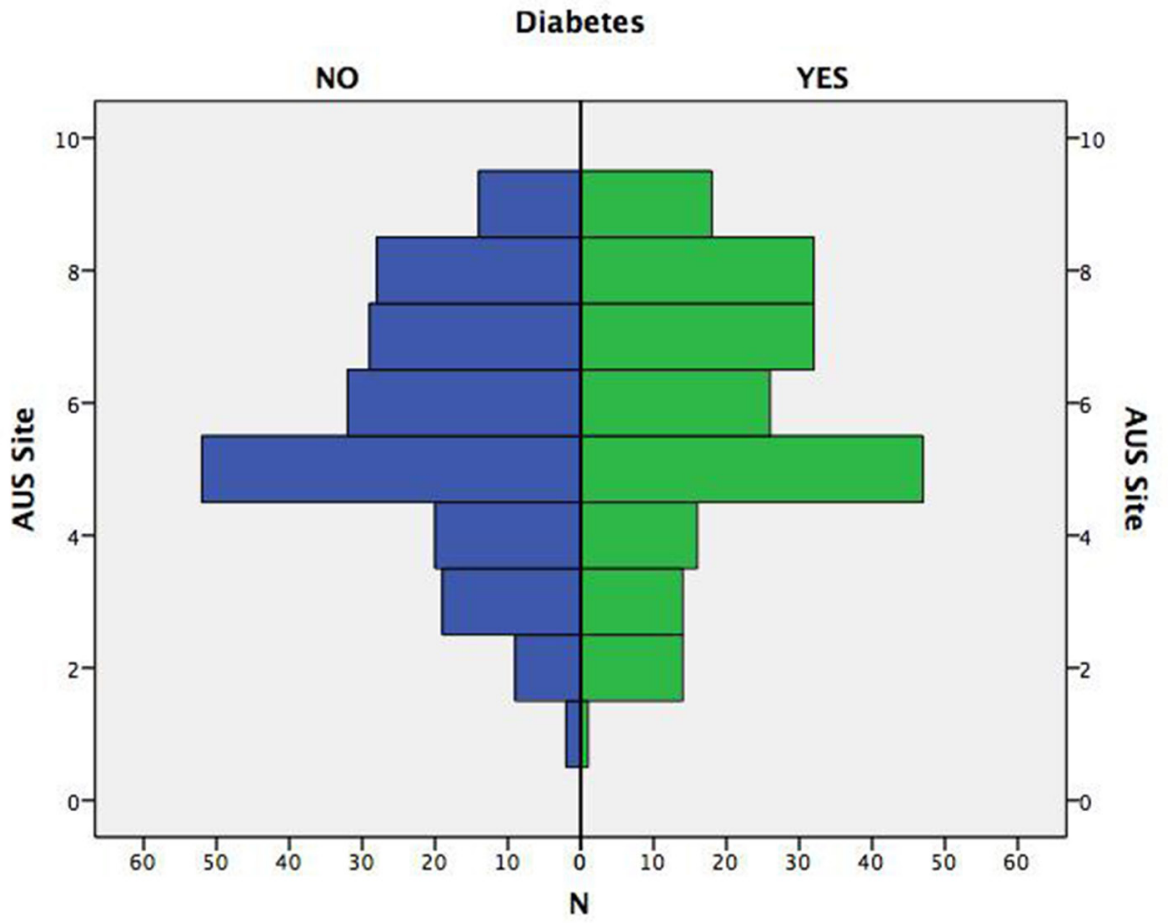

Figure 1 Histogram of appropriate use score according to site-reported coronary anatomy (AUS SITE $_{1}$ in patients with and without diabetes.

AUS $_{\mathrm{SITE}}$ and $\mathrm{AUS}_{\mathrm{CORE}}$ were significantly higher in patients who were evaluated by a heart team, suggesting multidisciplinary decision making is a surrogate of optimal revascularisation choice.

In patients with stable CAD, a functional testing strategy (used in $62 \%$ of patients) was far more common than an anatomical testing strategy with coronary CT angiography (used only for $8 \%$ of patients), in agreement with the neutral findings of the PROMISE (PROspective Multicenter Imaging Study for Evaluation of chest pain) trial. ${ }^{16}$ Notably, the use of functional testing was similar in patients with intermediate and with high PTP of significant CAD, suggesting that the determination of PTP, considered the first major step in clinical decision making in this patient population, ${ }^{7}$ has limited influence in the real world to define a diagnostic strategy. Also, 33\% of patients with stable CAD with unknown coronary anatomy had neither functional nor anatomical testing before PCI and only $38 \%$ had maximal anti-ischaemic medical therapy before PCI, proportions that have contributed to the observed suboptimal appropriateness and call for implementation of quality improvement initiatives. Indeed, it has been observed that most $(55.5 \%)$ of Medicare patients with stable CAD do not have documentation of ischaemia by non-invasive testing prior to elective PCI and that pre-PCI stress testing was associated with lower mortality in patients undergoing elective PCI. ${ }^{17} 18$ Overall, these findings suggest a need to focus on PTP assessment
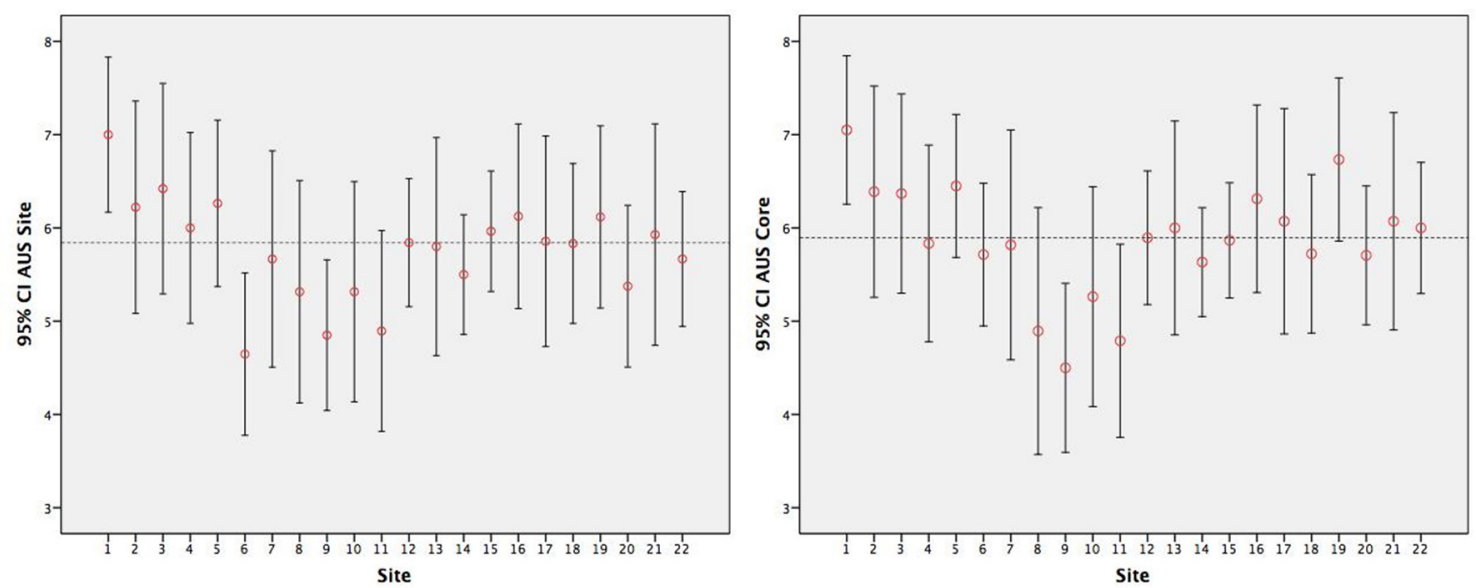

Figure 2 Error bars of AUS SITE $_{\text {(left) and AUS }}$ CORE (right) by participating sites. The dotted line indicates the median AUS SITE $_{\text {level }}$ (5.8). AUS, appropriate use score. 


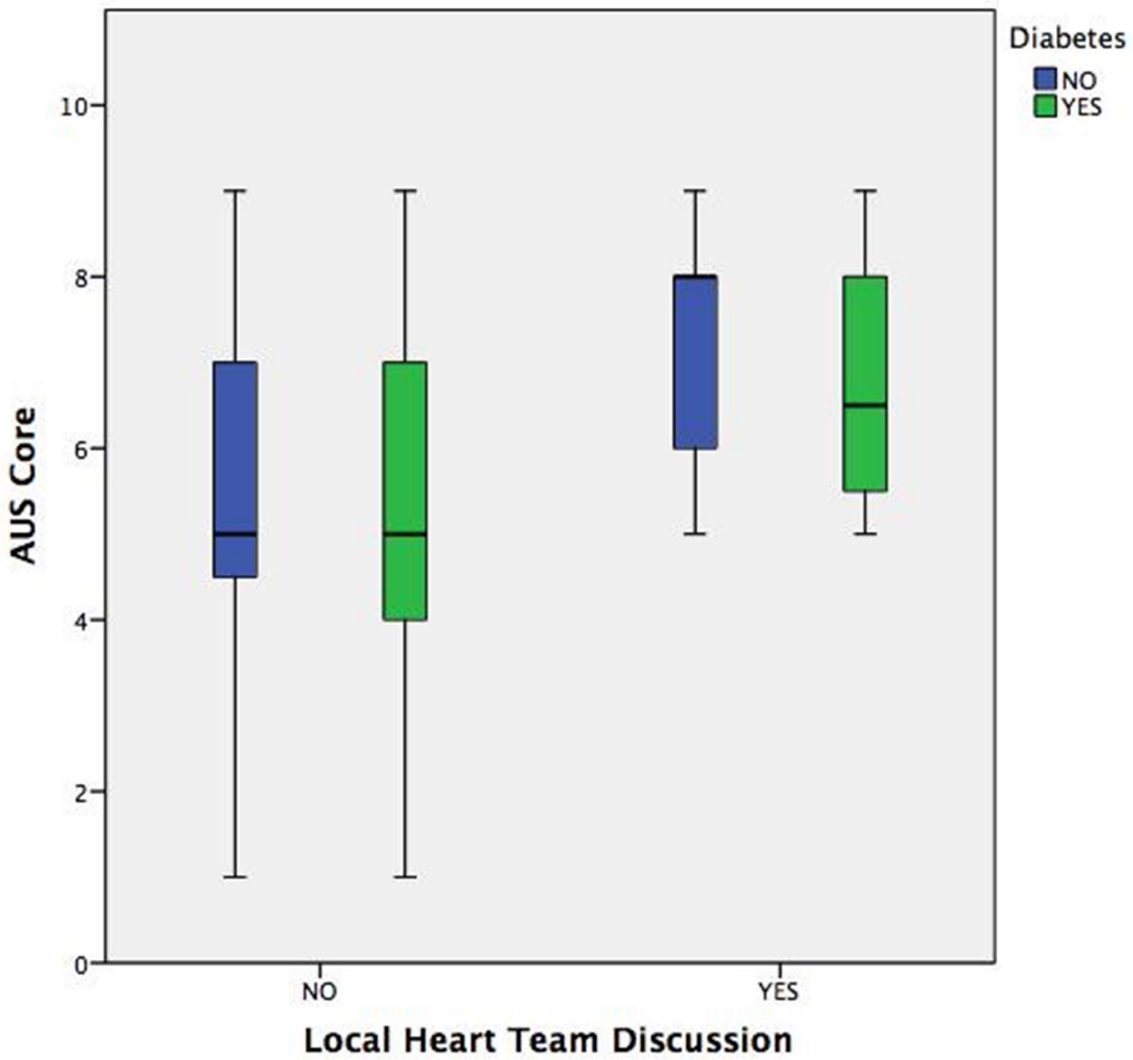

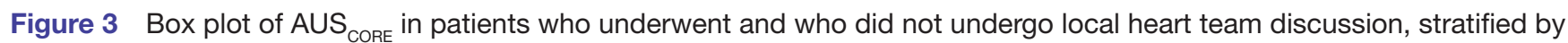
diabetes status. AUS, appropriate use score.

in decision making, appropriate classification of risk by non-invasive tests and optimisation of medical therapy before PCI.

\section{Adherence to and implementation of ESC guidelines}

To explore potential 'specialty bias'-that is, PCI treatment in patients with an indication for CABG-we examined the proportion of patients with complex coronary lesions, including significant disease of the left main, proximal LAD and/or three-vessel disease, who were treated ad hoc without evidence of heart team discussion in the medical charts. We observed that only 1 of 10 eligible patients underwent heart team discussion and $75 \%-80 \%$ of patients with complex coronary anatomy were treated ad hoc, with no significant differences in sites with and without on-site cardiac surgery. To further explore this, we surveyed investigators to better understand local decision-making processes. We observed that most sites did not have a written institutional protocol and decision of heart team convocation was left at the discretion of the interventional cardiologist on call. The high proportion of ad hoc PCI in patients with complex disease and the lack of structured local heart teams identify a substantial opportunity to improve multidisciplinary decision-making processes and indicate the need for standardised institutional protocols that (1) should avoid the need for the systematic case-by-case review of all diagnostic angiograms but guide the management of complex cases, (2) define standards for heart team composition and roles, and (3) generate consensus on practical ways to implement them.

The SYNTAX score is also considered by the guidelines to inform choice on optimal type of revascularisation. This score, which relies on subjective assessment of lesions using coronary angiography, is well known to have limited reproducibility. ${ }^{19}$ The highest kappa value observed in a study of the SYNTAX investigators to assess intraobserver variability was 0.54 , and only 0.36 for bifurcations ${ }^{19}$ and interobserver reproducibility was even lower. ${ }^{20}$ We therefore decided to adopt a conservative approach to define a disagreement between reviewers (arbitrarily defined as a difference of at least 10 points or change of tertile) but still observed a disagreement in $21 \%$ of patients. This variability suggests that for clinical decision making, SYNTAX score should not be used in isolation but rather integrated with clinical data. The SYNTAX II score was developed to address this need. By implementing this score in the APACHE population, we observed that in the vast majority of patients (84\%) either modality of revascularisation was recommended. However, in the 40 patients $(12 \%)$ where CABG was modality of choice, a heart team discussion was performed only in three cases.

FFR, the current gold standard for the functional assessment of lesion severity, ${ }^{21}$ is recommended (class I, level of evidence A) to identify haemodynamically relevant coronary lesions in stable patients when evidence of ischaemia is not available or to assess the functional consequences of 
moderate coronary stenoses. ${ }^{6}$ According to the ACL, the proportion of patients without documented ischaemia or borderline coronary stenoses who had an FFR-guided PCI was $25 \%$, although the absolute numbers were small ( 7 FFR in 28 patients). While recent data suggest that FFR use is increasing, these data indicate another gap in use of a well-established technique to define physiological consequences of a coronary stenosis, thus optimising appropriate indication for revascularisation.

Finally, we observed that prescription of guideline-directed medical therapy before PCI was suboptimal with just $23 \%$ of patients on 'optimal medical therapy' and a high prescription only for antiplatelet therapy before PCI $(>90 \%)$. This gap may have multiple reasons including resource availability, patient's compliance and physician preference, but should be an important, and easily modifiable target, for any quality improvement initiative. By quality, we intend the degree of match between healthcare services and the needs they are intended to meet. ${ }^{22}$ APACHE was designed to first quantify this match, inform the design of future investigations on this topic (including a planned larger initiative in Italy extended to the whole country designed to develop, implement and adhere to shared heart team protocols) and promote a continuous review of practice that may, in turn, inform a more effective, efficient and equitable resources allocation, and ultimately, better outcomes for patients.

\section{CONCLUSIONS}

Use of heart team approaches and adherence to guideline recommendation on coronary revascularisation in a real-world setting is limited. The APACHE study identifies substantial opportunities to improve the care of patients undergoing PCI.

\footnotetext{
Author affiliations

${ }^{1}$ Coronary Care Unit, Fondazione IRCCS Policlinico San Matteo, Pavia, Italy

${ }^{2}$ Cardiology, Ospedale Maggiore di Crema, Crema, Italy

${ }^{3}$ Cardiology, ASST Mantova-0spedale Carlo Poma, Mantova, Italy

${ }^{4}$ Cardiology, ASST Cremona-0spedale di Cremona, Cremona, Italy

${ }^{5}$ Cardiology, Ospedale Fornaroli di Magenta, Magenta, Italy

${ }^{6}$ Cardiology, ASST Bergamo Ovest-0spedale di Treviglio, Treviglio, Italy

${ }^{7}$ Cardiology, Azienda Ospedaliera di Desio e Vimercate, Vimercate, Italy

${ }^{8}$ Cardiology, Spedali Civili di Brescia, Brescia, Italy

${ }^{9}$ Cardiology, Ospedale di Circolo Fondazione Macchi, Varese, Italy

${ }^{10}$ Cardiology, Ospedale San Paolo, Milano, Italy

${ }^{11}$ Cardiology, Ospedali Riuniti Padova Sud Madre Teresa di Calcutta, Monselice, Italy

${ }^{12}$ Cardiology, Presidio Ospedaliero di Conegliano, Conegliano, Italy

${ }^{13}$ Cardiology, Presidio Ospedaliero di Cittadella, Cittadella, Italy

${ }^{14}$ Cardiology, ULSS 4 Alto Vicentino, Thiene, Italy

${ }^{15}$ Cardiology, IRCCS Policlinico San Donato, San Donato Milanese, Italy

${ }^{16}$ Cardiology, Ospedale San Gerardo, Monza, Italy

${ }^{17}$ Cardiology, Centro Cardiologico Monzino, Milano, Italy

${ }^{18}$ Cardiology, Fondazione Poliambulanza, Milano, Italy

${ }^{19}$ Cardiology, ASST Ovest Milanese, Legnano, Italy

${ }^{20}$ Cardiology, ASST di Lodi, Lodi, Italy

${ }^{21}$ Cardiology, Humanitas Research Hospital, Rozzano, Italy

${ }^{22}$ Cardiology, Ospedale di Padova, Padova, Italy

${ }^{23}$ Cardiology, Ospedale San Giovanni Evangelista, Tivoli, Italy

${ }^{24}$ Cardiology, Ospedale degli Infermi, Rivoli, Italy

${ }^{25}$ Cardiology, ASST-Papa Giovanni XXIII-Bergamo, Bergamo, Italy
}

${ }^{26}$ Cardiology, IRCCS MultiMedica, Milano, Italy

Correction notice /

Collaborators /

Contributors SL and SDS: study design, manuscript drafting, statistical analysis and critical revision. MM and GC: angiographic core lab analysis, critical revision. FM and DR: data management, statistical analysis and critical revision. CL, LBettari, MZ, PS, ST, MA, SG, CS, GP, AP, LP, LBassan, MBollati, PC, DT, MBrancati, AP, CP: critical revision. ML, GTarelli, GTarantini, LDL, FV: Central Heart Team and critical revision. GM: study design and critical revision.

Disclaimer /

Competing interests ICJME forms are available for all authors. SL reports honoraria for advisory boards from AstraZeneca, Daiichi Sankyo, and The Medicines Company during the conduct of the study outside the submitted work; LDL reports personal fees from Astra Zeneca, personal fees from Bayer, personal fees from Boehringer-Ingelheim, personal fees from Eli Lilly and Daiichi Sankyo, personal fees from Menarini, personal fees from The Medicines Company, outside the submitted work. SDS reports personal fees from Pfizer, personal fees from AstraZeneca, personal fees from Daiichi Sankyo, personal fees from Correvio, outside the submitted work. The other authors report nothing to disclose. All authors have read and understood BMJ policy on declaration of interests and have no other relevant interests to declare in addition to these.

Patient consent Obtained.

Ethics approval Fondazione IRCCS Policlinico S Matteo (Central IRB) and IRBS from 21 all sites involved.

Provenance and peer review Not commissioned; externally peer reviewed.

Data sharing statement № additional unpublished data for this study will be available.

Open Access This is an Open Access article distributed in accordance with the Creative Commons Attribution Non Commercial (CC BY-NC 4.0) license, which permits others to distribute, remix, adapt, build upon this work non-commercially, and license their derivative works on different terms, provided the original work is properly cited and the use is non-commercial. See: http://creativecommons.org/ licenses/by-nc/4.0/

(C) Article author(s) (or their employer(s) unless otherwise stated in the text of the article) 2017. All rights reserved. No commercial use is permitted unless otherwise expressly granted.

\section{REFERENCES}

1. Chan PS, Rao SV, Bhatt DL, et al. Patient and hospital characteristics associated with inappropriate percutaneous coronary interventions. J Am Coll Cardiol 2013;62:2274-81.

2. Baig SS, Altman DG, Taggart DP. Major geographical variations in elective coronary revascularization by stents or surgery in England. Eur J Cardiothorac Surg 2015;47.

3. Patel MR, Dehmer GJ, Hirshfeld JW, et al. ACCF/SCAI/STS/AATS/ AHA/ASNC/HFSA/SCCT 2012 Appropriate use criteria for coronary revascularization focused update: a report of the American College of Cardiology Foundation Appropriate Use Criteria Task Force, Society for Cardiovascular Angiography and Interventions, Society of Thoracic Surgeons, American Association for Thoracic Surgery, American Heart Association, American Society of Nuclear Cardiology, and the Society of Cardiovascular Computed Tomography. J Am Coll Cardiol 2012;59:857-81.

4. Desai NR, Bradley SM, Parzynski CS, et al. Appropriate Use Criteria for Coronary Revascularization and Trends in Utilization, Patient Selection, and Appropriateness of Percutaneous Coronary Intervention. JAMA 2015;314:2045-53.

5. Maier W, Enderlin MF, Bonzel T, et al. Audit and quality control in angioplasty in Europe: procedural results of the AQUA Study 1997: assessment of 250 randomly selected coronary interventions performed in 25 centres of five European countries. AQUA Study Group, Nucleus Clinical Issues, Working Group Coronary Circulation, of the European Society of Cardiology. Eur Heart J 1999;20:1261-70.

6. Windecker S, Kolh P, Alfonso F, et al. 2014 ESC/EACTS Guidelines on myocardial revascularization: The Task Force on Myocardial Revascularization of the European Society of Cardiology (ESC) and the European Association for Cardio-Thoracic Surgery (EACTS) Developed with the special contribution of the European Association 
of Percutaneous Cardiovascular Interventions (EAPCI). Eur Heart $J$ 2014;35:2541-619.

7. Montalescot G, Sechtem U, Achenbach S, et al. 2013 ESC guidelines on the management of stable coronary artery disease: the Task Force on the management of stable coronary artery disease of the European Society of Cardiology. Eur Heart J 2013;34:2949-3003.

8. Bonzel T, Schächinger V, Dörge H. Description of a Heart Team approach to coronary revascularization and its beneficial long-term effect on clinical events after PCl. Clin Res Cardiol 2016;105:388-400.

9. Nallamothu BK, Tommaso CL, Anderson HV, et al. ACC/AHA/SCAI/ AMA-Convened PCPI/NCQA 2013 performance measures for adults undergoing percutaneous coronary intervention: a report of the American College of Cardiology/American Heart Association Task Force on Performance Measures, the Society for Cardiovascular Angiography and Interventions, the American Medical AssociationConvened Physician Consortium for Performance Improvement, and the National Committee for Quality Assurance. J Am Coll Cardiol 2014;63:722-45.

10. Farooq V, van Klaveren D, Steyerberg EW, et al. Anatomical and clinical characteristics to guide decision making between coronary artery bypass surgery and percutaneous coronary intervention for individual patients: development and validation of SYNTAX score II. The Lancet 2013;381:639-50.

11. Shahian DM, O'Brien SM, Filardo G, et al. The Society of Thoracic Surgeons 2008 cardiac surgery risk models: part 1--coronary artery bypass grafting surgery. Ann Thorac Surg 2009;88(1 Suppl):S2-S22.

12. Nashef SA, Roques F, Sharples LD, et al. EuroSCORE II. Eur J Cardiothorac Surg 2012;41:734-45.

13. Tonino PA, De Bruyne B, Pijls NH, et al. Fractional flow reserve versus angiography for guiding percutaneous coronary intervention. N Engl J Med 2009;360:213-24.
14. Hulley SB, Cummings SR, Browner WS, et al; Designing clinical research: an epidemiologic approach. 4th ed. Philadelphia, PA: Lippincott Williams \& Wilkins, 2013.

15. Patel MR, Dehmer GJ, Hirshfeld JW, et al. ACCF/SCAI/STS/ AATS/AHAVASNC 2009 Appropriateness Criteria for Coronary Revascularization: a report by the American College of Cardiology Foundation Appropriateness Criteria Task Force, Society for Cardiovascular Angiography and Interventions, Society of Thoracic Surgeons, American Association for Thoracic Surgery, American Heart Association, and the American Society of Nuclear Cardiology Endorsed by the American Society of Echocardiography, the Heart Failure Society of America, and the Society of Cardiovascular Computed Tomography. J Am Coll Cardiol 2009;53:530-53.

16. Douglas PS, Hoffmann U, Patel MR, et al. Outcomes of anatomical versus functional testing for coronary artery disease. N Engl J Med Overseas Ed 2015;372:1291-300.

17. Lin GA, Dudley RA, Lucas FL, et al. Frequency of stress testing to document ischemia prior to elective percutaneous coronary intervention. JAMA 2008;300:1765-73.

18. Lin GA, Lucas FL, Malenka DJ, et al. Mortality in Medicare patients undergoing elective percutaneous coronary intervention with or without antecedent stress testing. Circulation 2013;6:309-14.

19. Garg S, Girasis C, Sarno G, et al. SYNTAX trial investigators. The SYNTAX score revisited: a reassessment of the SYNTAX score reproducibility. Catheter Cardiovasc Interv 2010;75:946-52.

20. Serruys P, Onuma Y, Garg S, et al. Assessment of the SYNTAX score in the syntax study. Eurolntervention 2009;5:50-6.

21. Pijls NHJ, de Bruyne B, Peels K, et al. Measurement of fractional flow reserve to assess the functional severity of coronary-artery stenoses. N Engl J Med Overseas Ed 1996;334:1703-8.

22. Berwick DM. Avoiding overuse-the next quality frontier. Lancet 2017;390:102-4. 